\title{
A RARE CASE OF A SOLITARY, LARGE PANCREATIC DUCT CALCULUS IN A YOUNG NON ALCOHOLIC MALE
}

\author{
T. R. Ravi Mohan'1, D. Keertana², B. Moksha Prasuna3 ${ }^{3}$ Ch. Madhusudhan4, R. Prathap Reddy5
}

\section{HOW TO CITE THIS ARTICLE:}

T. R. Ravi Mohan, D. Keertana B. Moksha Prasuna, Ch. Madhusudhan, R. Prathap Reddy. "A Rare Case of a Solitary, Large Pancreatic Duct Calculus in a Young Non Alcoholic Male". Journal of Evolution of Medical and Dental Sciences 2015; Vol. 4, Issue 29, April 09; Page: 5082-5084, DOI: 10.14260/jemds/2015/742

INTRODUCTION: Tropical pancreatitis is a non-alcoholic form of idiopathic, chronic, calcifying pancreatitis observed in young adults in tropical countries. Multiple and large intraductal calculi are a hallmark of the disease. We report a case of a 29 year old male patient with a large, solitary pancreatic duct stone who was managed successfully with lateral pancreaticojejunostomy. This case is reported in view of rarity of presence of single, large calculus in tropical pancreatitis.

CASE REPORT: A male patient aged 29 years presented with complaints of episodes of abdominal pain since past 6 months. The pain was intermittent, located in the epigastrium, radiating to the back and dull aching in nature. There was no history of nausea, vomiting, or altered bowel habits. He revealed that he has been consuming alcohol for the past 3years, but it was limited to 1 to 2 beers per week. There was no family history of similar condition.

Examination revealed a thin built man, with no clinical evidence of jaundice. Abdominal examination showed no remarkable findings. Rest of physical examination was normal.

Patient was investigated which showed normal Hb, normal blood sugar level, normal serum amylase, renal function tests and liver function tests.

Endoscopic ultrasound showed altered echotexture in the pancreas with heterogenous foci and calcifications with main pancreatic duct dilated to $1.3 \mathrm{~cm}$. A solitary calculus was seen in the MPD in the region of the head of pancreas measuring $4.5 \times 1.5 \mathrm{~cm}$. Gall bladder and liver appeared normal.

CT abdomen showed pancreas to be atrophic with a $4.2 \mathrm{~cm}$ dense calculus in head and neck with dilated MPD. Illdefined patchy hypodense areas were seen in the tail and peripancreatic regions.

MRCP showed a $4.3 \times 1.1 \mathrm{~cm}$ calculus in the MPD in the head and neck and MPD dilated to $13 \mathrm{~mm}$ in body and tail.

Patient was taken up for laparotomy on 25/08/2012.

The MPD was found to be dilated to around $1 \mathrm{~cm}$. A $5.5 \times 1.5 \mathrm{~cm}$ solitary calculus was found in the head and neck region of pancreas and was delivered.

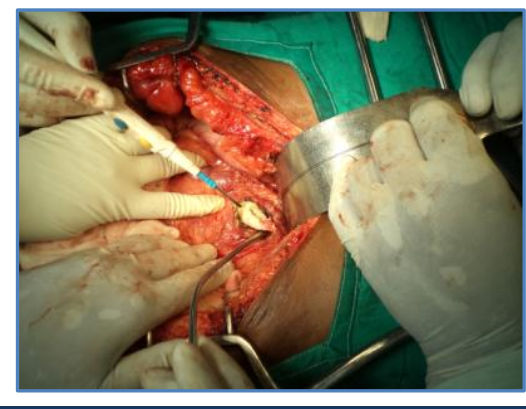

Fig. 1: MPD being opened over the stone

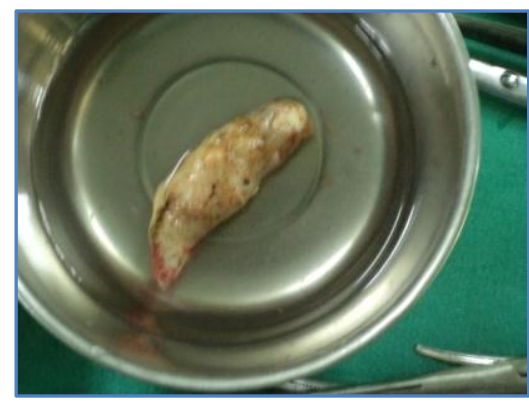

Fig. 2: Pancreatic duct calculus 


\section{CASE REPORT}

Roux-en-Y loop of jejunum was mobilized and anastamosed with the pancreatic duct. Patient had an uneventful recovery and was discharged on 7 th post-operative day.

DISCUSSION: Tropical chronic pancreatitis (TCP) is a form of chronic calcific non-alcoholic pancreatitis, seen almost exclusively in the developing countries of the tropical world. Some of the distinctive features are younger age at onset, presence of large intraductal calculi, more aggressive course of disease and a high susceptibility to pancreatic cancer.[1] The prevalence of tropical pancreatitis is about $126 / 100000$ population in southern India.[2]

Clinically, $>90 \%$ of patients present with abdominal pain. About $25 \%$ of patient develop diabetes mellitus which generally requires insulin for its control but is ketosis resistant. [2] The diabetic stage of tropical chronic pancreatitis is referred to as Fibrocalculous Pancreatic Diabetes(FCPD). The presence of SPINK1 mutations in both TCP and FCPD suggests a common genetic basis.[3]

Mahurkar et al, suggested the two hit model for the pathogenesis of TCP. First hit is likely to be loss of balance between activation events and degradation of active trypsin, leading to presence of persistent 'super trypsin' within the acinar cell, resulting in inflammation. Presence of additional genetic or environmental factors constitute the second hit. ${ }^{[4]}$ According to Sathiraj E, malnutrition is not an etiological factor of TCP and weight loss occured as a result of low calorie intake after the onset of TCP.[5]

Pancreatic calculi are the hallmark of the disease. They may be solitary or multiple and sometimes the entire pancreas may be studded with calculi. The stones tend to be large, dense and rounded with well-defined edges in contrast to the small, speckled, ill-defined stones in alcoholic chronic pancreatitis.[1]

According to Vinaya Kumar et al, over the last three decades there has been a considerable change in the natural history of the disease and it now affects an older population and has become less severe. Diabetes mellitus is mild and is easier to control, but the microvascular complications have become prominent. Carcinoma pancreas has emerged as a leading complication and the most important cause of mortality.[7]

Indications of surgery in TCP include: severe, intractable pain, pancreatitis associated complications of adjacent organs, distal common bile duct stenosis, duodenal stenosis, segmental portal hypertension, pancreatic pseudocyst with ductal pathology, internal pancreatic fistula and pancreatic ascites, exclusion of malignancy despite extensive workup, progressive destruction of the organ despite conservative treatment and, occasionally, progressive ill health and problems arising from malnutrition. ${ }^{[6]}$

Patients with tropical chronic pancreatitis who undergo the Puestow procedure not only have relief from pain but also improvement of diabetes. ${ }^{[8]}$ Ductal decompression can also be achieved by endoscopic techniques. Small pancreatic calculi can be extracted using a basket after an endoscopic pancreatic sphincterotomy. This technique may not be successful for large stones in the MPD. The problem can be overcome by fragmenting the calculi. ESWL is an established modality in the management of large pancreatic duct calculi.[9]

\section{REFERENCES:}

1. K. Barman, G. Premalatha, V. Mohan. Tropical chronic pancreatitis, Postgrad Med J. Nov 2003; 79(937): 606-615. 
2. Tandon RK, Garg PK. Tropical pancreatitis. Dig Dis 2004; 22(3): 258-266.

3. Chakraborty Monali. An insight into the etiology of tropical chronic pancreatitis and fibrocalculous pancreatic diabetes. International Research Journal of Medical Sciences, March 2013; Vol 1 (2): 5-14.

4. Swapna Mahurkar, D. Nageshwar Reddy, G. Venkat Rao, Giriraj Ratan Chandak. Genetic mechanisms underlying the pathogenesis of tropical calcific pancreatitis. World J Gastroenterol, 2009 Jan 21; 15 (3): 264-269.

5. Sathiaraj E, Gupta S, Chuthe M, Mahurkar S, Mansand MJ, Rao GV, Reddy DN. Malnutrition is not an etiological factor in the development of tropical pancreatitis-a case control study of southern indian patients.Trop Gastroenterol,2010 July-Sep; 31(3); 169-74.

6. Mihir K. Mohapatra. Surgical management of tropical calcific pancreatitis-observations from Orissa. Chronic pancreatitis and pancreatic diabetes in India.2004; 26: 340-347.

7. Vinaya Kumar KR, Biju Lal. Tropical P Ancreatitis-changing trends. Chronic pancreatitis and pancreatic diabetes in india.2004; chp 11: 127-136.

8. Sandeep S sidhu, Samiran Nundy, Rakesh K Tandon. The effect of modified Puestows procedure on diabetes in patients with tropical chronic pancreatitis-a prospective study. The American Journal of Gastroenterology, 2001; 96: 107-111.

9. Manu Tandon, D. Nageshwar Reddy, Darishetty Santosh, Koppoju Vinod, Mohan Ramchandani, Gupta Rajesh, Kotla Rama, Sandeep Lakhtakia, Rupa Banerjee, Nitesh Pratap, Guduru Venkat Rao. ESWL and endotherapy for pancreatic calculi-a large single center experience. Indian J. Gastroenterol 2010; 29: 143-148.

\section{AUTHORS:}

1. T. R. Ravi Mohan

2. D. Keertana

3. B. Moksha Prasuna

4. Ch. Madhusudhan

5. R. Prathap Reddy

\section{PARTICULARS OF CONTRIBUTORS:}

1. Assistant Professor, Department of Surgical Gastroenterology, Osmania Medical College and hospital.

2. Resident, Department of Surgical Gastroenterology, Osmania Medical College and hospital.

3. Assistant Professor, Department of General Surgery, Osmania Medical College and hospital.

FINANCIAL OR OTHER COMPETING INTERESTS: None
4. Associate Professor, Department of Surgical Gastroenterology, Osmania Medical College and hospital.

5. Professor, Department of Surgical Gastroenterology, Osmania Medical College and hospital.

\section{NAME ADDRESS EMAIL ID OF THE CORRESPONDING AUTHOR:}

Dr. T. R. Ravi Mohan,

\# G2, Woodswale,

Adjacent Allahabad Bank,

Madhura Nagar, Hyderabad,

Telangana-500038.

E-mail: ravimohantr@gmail.com

Date of Submission: 11/03/2015.

Date of Peer Review: 12/03/2015.

Date of Acceptance: 25/03/2015.

Date of Publishing: 09/04/2015. 